changement apporté à l'un des facteurs de l'équilibre, pression ou température, entraîne une transformation du système qui lend à produre un changement de signe contraire du facteur considéré. Jamais l'on a observé de faits en désaccord avec les lois du déplacement de l'équilibre, à condition, toutefois, de se souvenir que quand la thermodynamique indique qu'un phénomène ne peut se produrre, il ne se produrt réellement pas, ma1s que quand elle indique qu'il peut se produire, 11 arrive souvent qu'il ne se produit pas C'est en cela que réside la cause de la longue période de recherches qui a précédé la découverte des loss de Van'T Hoff et de Le Chatelier; aux températures ordinaires, en effet, les équilıbres observés ne sont souvent pas des équilibres véntables, et alors les lois du déplacement de l'équilibre ne s'appliquent plus.

Que peut-on conclure de cet exposé rapide des conséquences des principes de Mayer et de Carnot-Clausius, sinon que la Thermodynamique vient complèter la Mécanique et faire sortir la Chimie dé la période prémathémamique, par laquelle débute toute science.

J. LAHOUSSE,

Professeur à l'Ecole Centrale Lyonnaise

\section{UTILISATION DES MARÉES}

Le charbon que l'on tire de la terre ne se renouvelant pas au fur et à mesure de son extraction, il arrivera fatalement un moment où ce charbon viendra à manquer. Ce moment est évidemment très éloigné, mais, blen avant qu'il n'arrive, le prix du charbon aura certainement aug. menté dans d'assez grandes proportions. Aussi, les pays essentiellement industriels doivent-ils se préoccuper, d'ores et dejà, de rechercher un moyen qui puisse leur permettre de maintenir chez eux leur developperrent industriel. Telle est la thèse qu'a développée M. I. SAUNDERs, dans l'Engtneering Review, où il a montré que l'utilisation des marées peut produrre des quantités considérables d'énergio, ear, si la chute est faible, le débit, par contre, est considérable.

L'intervalle qui existe enlre deux marées est d'environ 12 heures 24. minutes, et, pour chaque révolution de la lune autour de la terre, il existe deux époques correspondant respectivement à un maximum et à un minimum de l'amplitude de la marée. Celle-ci varie considérablement le lons. dos côtes anglaises, et elle atteint son maximum à Bristol avec 40 pieds (12m192). A Southampton et à Douvres, les fortes marées sont, respectivement, de 13 et $181 / 2$ pieds, et les plus faibles de $91 / 2$ et 15 pieds.

On avait bien déjà songé, depuis longtemps, à utiliser les marées en recueillant l'eau de mer dans de vastes réservoits lorsque la mer est haute, et en faisant passer l'eau du réservoir à travers des turbines, pour la renvoyer à la mer lorsque celle-ci est hasse $\left(^{*}\right)$; malheureusement, on s'cst heurté à un grave inconvénient: la puissance de la chute ainsi utilısée n'était point constante, et, bien au contraire, elle était éminemment variabłe. Cet obstacle aurait rendu lutilisation des marées impraticable, si un ingénieur français des Ponts-et-Chaussées, M. DEcocon, n’avait su houver un procédé permettant de tourner la difficulté, et de vendre la puissance produite sensiblement constante ainsi qu'on va le voir.

Co dispositif consiste à emmagasiner l'eau de mer, à marée haute, dans un premier réservoir, dit réservoir supérieur, puss à envoyer cette eau, ainsi emmagasinée, dans un second réservoir, dit réservoir inférieur, après l'avoir fait pusser dans des turbines pour produire de lénergie électrique. Ce second réservoir est mis en communication avec la mer à marée basse.
Le mouvement de l'eau dans les hassins est graphiquement représenté par la figure 1. ACA'C représente les variations du niveau de la mer sous l'influence de la marée. Lorsque la maréc esta à son plein, en $\mathrm{A}$, on ferme los orifices d'introduction de l'eau de mer dans le réservoir supérieur. L'usine hydraulique fonctionnant, le niveau baisse progressivement dans ce réservoir supérieur, tandis qu'il

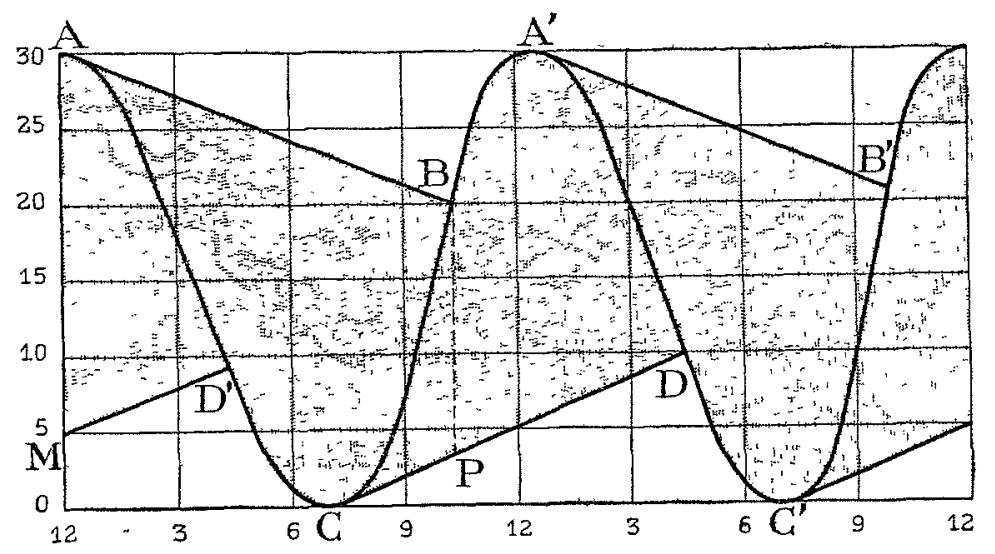

Fra. 1. - Graphique représentint le mouvement de leau daus les réservorrs

monte dans le réserroìr inférieur. Lorsque le niveau de l'eau, dans ce dernier bassin, est arrivé à la hauteur de celui de la mer, en $D^{\prime}$, on ouvre les orlfices de communicatıon du réservoir inférjeur avec la $\mathrm{mer}^{2}$, et commo celle-ci continue à baisser, le niveau du réservoir inlérleur baisse aussi. Lorscue la marée est arrivée à son point le plus bas, en $\mathrm{C}$, on ferme les orifices de communication du reservoir inférieur; alors l'eau s'y élève peu à peu, jusqu'en $\mathrm{P}$, tandis que la mer remonte el que le niveau du réservoir supélieur continue à diminuer. Lorsçue le niveau do ce réservoir ar. rive à la hauteur de celui de la mer, en $B$, on ouvre les orifices de communication du réservoir supérieur, et le mvéau de l'eau, daus ce réservoir, monte alors avec celui de la mer, jusqu'en A', tandis que celui du réservoir inférieur contiuue à s'élever jusqu'en $\mathrm{D}$. On a ainsi réalısé un cycle complet. Puis on recommence les mêmes opérations, et ainsi de suite, indéfniment.

La quantité d'eau considérable qui entre on jeu dans une pareille installation exige de nombreuses vannes de grandes dimensions. La manceuvre de ces vannes demande toujours un certain temps, mais cela n'est pas une gêne à cause de la lenteur du mouvement de la marée.

La hauteur de chule n'est pas tout-à fait constante, néanmoins, sa variation est relativement faible: sa plus pelite valeur est égale à $\mathrm{BP}$ et sa plus grande valeur à $\mathrm{AM}$; ces deux valeurs extrêmes different, en somme, assez peu l'une de lautre. La puissance peut donc être maintenue constante en faisant varier légèremont le débit aljsorbé par les moteurs hydrauliques.

M. Saunders signale trois projets d'utilisation dos marées basés sur ce procédé.

A Chichester, le port serait divisc en deux parties sensiblement égales, au moyen dun barrage en maconnerie sur lequel serait établie l'usine hydro-électrique. Ce barrage serait continué par une digue en terre, et un barrage en maçonnerie séparerait les deux réservoirs d'avec la mer, ainsi que le montre la figure $\_$ci-jointe.

L'usine gónćratrice comporterait 8 turbines quadruples, a axe vertical, de 850 chevaux chacune, chaque groupe de 4 turbines actionnant directement un alternateur triphasé. On pourrait ainsi produire 6800 chevaux au moment des petites marées. Au moment des fortes maréos, on pourrait encore produire 7000 autres chevaux pendant dix heures par jour ; mais, comme cette puissance est irrégulière, elle a beaucoup moins de valeur marchande; si l'on en tient comple, quand même, la puissance moyenne de la marée 
ressort a 8000 chevaux de 24 heures. M. Saunders estime que le cheval peut très bien se vendre 112,5 francs $(4,5 £)$; en comptant 12 pour 100 pour les dépenses d'exploitation, l'intérêt et l'amortissement (ce qui est peu), le prix du cheval aménagé pourrait être de 938 francs, et la dépense de premier établissement ressortirait pour cette installation a 7,5 million: de francs.

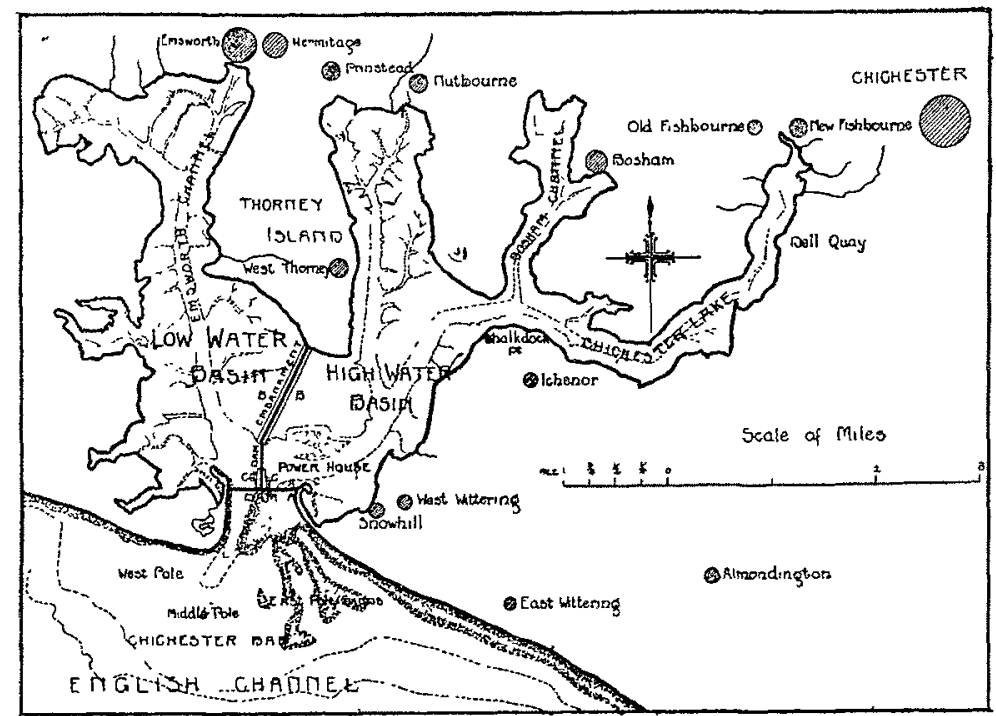

Fig. 9. - Aménagement du port de Chichester.

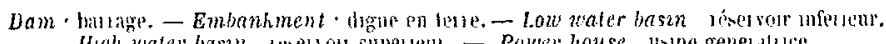

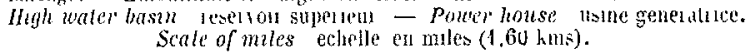

Un autre exemplo cité est celui du Menai Strait (détroit do Menai). On barrerait le détroit à chacune de ses extrémutés, et vers son milieu, de manière à créer deux réservoirs. L'usine génératrice serail élablie sur le barrage du milieu. Les hauteurs des marées ne sont pas les mêmes aux deux extrémités : à Beaumaris, du côté est, où se trouverait la prise d'eau du hassin supérieur, la hauteur des fortes marées est de 23 pieds, ct celle des pelites marées de 16 pieds 1/2, tandis qu'à Carnarvon Bar, à l'ouest, où se trouverait la sortie de l'eau du bassin inférieur, ces hauleurs ne sont que de $15,3 / 4$ et 12 pieds. La puissance produite serait de 13500 chevaux, au moment des petıtes marées, augmentés de 15000 chevaux pendant 10 heures aux moments des fortes marées du printemps; la puissance moyenne rescorlant à 14500 chevaux de 24 heures. En comptant le cheval au nême urix que précédemment, la dépense pourrait atleindre 13,5 millions.

Les barrages des extrémlés, ainci que celui du milieu, seraient munis d'écluses pour assurer le service de la navigation.

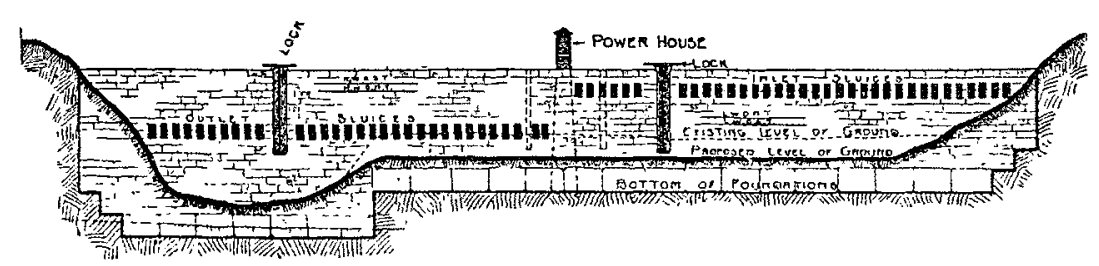

Fia. 3. - Fisure montrant la disposition des orifices dadmission (unlet sluices) ct déracuation (oullet sluices) de leau de mer. Lerel of around : mea du than - Loch ectuse.

Enfin, un troisième cxemple cité est celui du canal de Bristol. Le Jassin supérieur serait constitué par l'embouchure de la liviere Severn, et le bassin inférieur par des marais, isolés de la mer au moyen de digues, La chute maxima serat de 30 pieds, et la chute minima de 17 pieds ; la chule moyenne, pendant les 24 heures, serait de 20 pieds $\left(6^{\mathrm{m}} 096\right)$. La puissance produite serait ici de 210000 chevaux en petites malées, et en tenant compte des $15000 \mathrm{~m}^{3}$ débjtés par seconde par la rivière Severn.
Pendant les fortes marées du printemps, celte puissance serait encore accrue de 300000 chevaux pendant 10 heures, cequi correspondrait, en moyenne, à la puissance formidable de 260000 chevaux pendant 24 heures. En comptant le cheval toujours au même prix, on pourrait rémunérer l'énorme capital de 244 millions de francs, représentant à peu près la dépense de promier établissement $\left(^{*}\right)$.

Si l'on se reporte au dessin de la fig. l, qui représente précisément le mouvement de la marée à l'entrée du canal deBrisjol, on volt que la plus grande dénivellation des eaux, dans le bassin supérieur, n'est que de 10 pieds en petites marées, au lieu de 30 qu il est actuellement. La navigalion, dans le canal serait donc, de ce chef, considérablement améliorée.

Avec le nouveau procédé de M. Decoeur, la légère varia. tion qui subsiste dans la hauteur de la chute riest plus un inconvénient bien grave, car un certain nombre dinstallations à basses chutes fonctionnent avec des valiations parfois considérables. M. Saunders cite notamment le canal de Jonage, à Lyon, où la chule varie de 26 à 33 piels; a Schaffouse, cette variation est de 11,6 à 14,8 pieds; $\dot{a}$ Rheinfelden, elle est de 14,6 à 16 pieds.

Si l'on établit l'installation seulement pour le niveau des petites marées. on peut réaliser, toute l'année, une puissance constante et assurer un service public. Si l'on fait do l'électro-chimie, on pourra, bien souvent, utiliser le supplé-

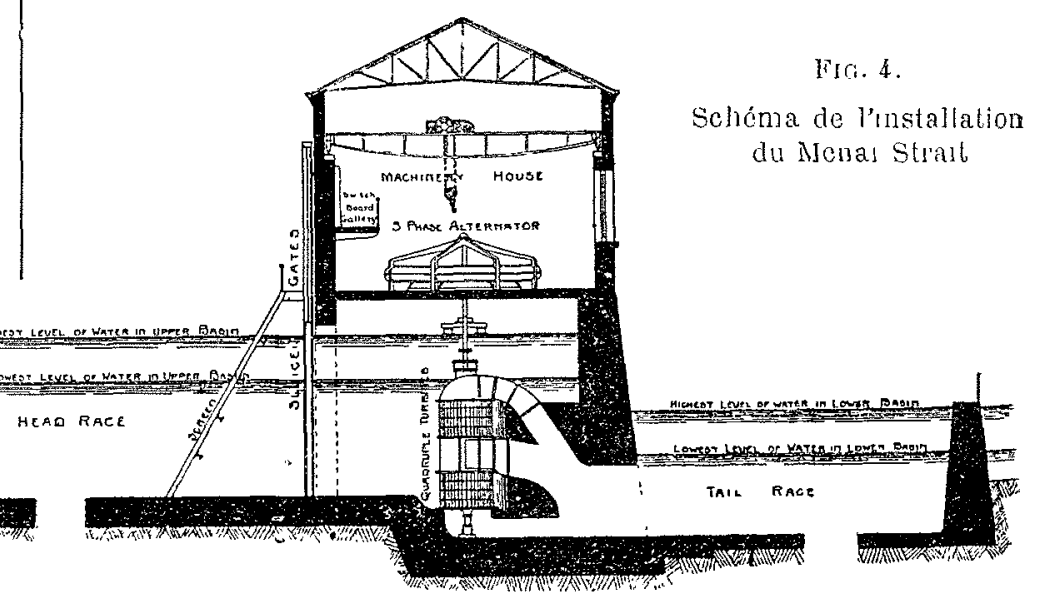

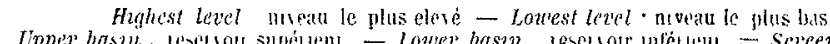

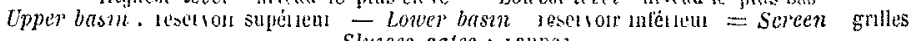

ment de puissance (les chevaux périodiques) fourni par l'augmentation de l'amplitude aux moments des fortes maréss; on ralentira simplement la fabrication au moment des basses eaux. Lt, ici, l'on n'a pas à craindre que la limile du débit ne vienne á tendre vers zéro, comme cela a été sur le point de se produire pour trop de cour's d'eau, en cette anrée 1906 qui a été exceptionnellement sèche. Si la: chute varie, le débit reste constant.

En résumé, l'utilisation des marées peut permettre de produire des quantités considérables d'énergie absolument inconnues dans nos Alpes, les 240000 chevaux, du canjal de Bristol, en petites marées, en sont la preuve. Et, si l'on se contente de chiffres plus modestes, on trouvera certainement bon nombre de baies (ou de fjords), analorues à celle du port de Cluchester, qui permettront de créer économiquement les immenses réservoir's nécessaires á l'utilisation des marécs, devenue close pratique.

$$
\text { M. P. }
$$

(*) Sur cette somme, 122 millions, soit la montie, seruenl consacrés à ćtabhr un barrage, en cimenl armé, de 30 kns de longucur, pout créer Je Jassin inférieur dout la suriace dte seratt pas nouns de 90 kilomètres carrés. 